Therapie bei LADA

\section{Alles eine Frage des Diabetes-Typs}

- Nicht nur die Prävalenz von Diabetes Typ 2 (T2D), sonder auch die von Typ-1-Diabetes (T1D) steigt, und zwar um etwa 4\% pro Jahr. Prof. Anette-Gabriele Ziegler, München, wünscht sich deshalb auch eine stärkere Konzentration auf diese Form der Erkrankung und rief zur vermehrten Aufklärung.

Eine Differenzierung zwischen Typ-1- und Typ2-Diabetes ist oft nicht einfach. T1D ist bekanntlich eine Autoimmunerkrankung, die eine Zerstörung der Betazellen und damit einen zunehmenden Insulinmangel bewirkt, während bei T2D primär eine Insulinresistenz vorliegt. Eine weitere Form ist der LADA (latent insulinpflichtiger Diabetes mellitus im Erwachsenenalter)-Diabetes. Dieser ist ebenfalls autoimmungbedingt und dem Formenkreis des T1D zuzuordnen. Außer einem absoluten Insulinmangel kann es zusätzlich zu Insulinresistenz kommen.

Da das Krankheitsbild bei T1D und T2D oft sehr ähnlich ist, kann bei Verdacht auf T1D oder bei entsprechender Familienanamnese ein ImmunScreening hilfreich sein. Finden sich dabei Antikörper gegen Insulin (IAA), Glutamatdecarboxylase (GADA) oder Thyrosinphosphatase (IA2A), so ist ein zukünftiger oder neumanifester T1D wahr- scheinlich. Auch die Analyse von Begleiterkrankungen könne aufschlussreich sein, sagte Ziegler. So sei T1D z.B. mit Hashimoto-Thyreoiditis, Zöliakie oder perniziöser Anämie assoziiert, T2D vornehmlich mit arterieller Hypertonie, Fettstoffwechselstörungen und Adipositas.

Langzeitstudien zeigen über Jahre hinweg stabile $\mathrm{HbA}_{1 \mathrm{c}}$-Werte für Typ-I-Diabetiker unter intensivierter konventioneller Insulintherapie (ICT), was laut Ziegler beweise, dass dies die richtige Therapieform für dieses Patientenkollektiv ist. Die Therapie des LADA gestalte sich hingegen schwieriger: Ziegler empfiehlt die Gabe von anfangs 2 x 500mg Metformin zusätzlich zu einer ICT; gleichzeitig könne man gegebenenfalls eine Insulinreduktion von etwa 10\% der Langzeit-Insulinmenge vornehmen. Das Problem dabei sei jedoch, dass Metformin bislang ausschließlich zur Therapie des T2D zugelassen ist. Der Einsatz bei T1D ist somit off-label und obliegt dem Risiko des Arztes - ein Umstand, der sich nach Ansicht Zieglers möglichst bald ändern sollte.

Quelle: Kolloquium „DiaLoQ - Im Fokus: Typ 1“, Februar 2011 in Potsdam, Veranstalter: Berlin-Chemie

Kurz notiert

\section{Add-on mit Sitagliptin: effektiv und mit weniger Hypoglykämien}

Als Kombipartner für Metformin ist der DPP-4-Hemmer Sitagliptin (Januvia ${ }^{\circledast}$ ) dem Sulfonylharnstoff (SH) Glimepirid nicht unterlegen: Die Blutzuckerkontrolle von Typ-2-Diabetikern war in einer aktuellen Studie bei beiden Therapieregimen ähnlich [1], allerdings gab es mit Sitagliptin signifikant weniger Hypoglykämien und eine bessere Gewichtskontrolle. In der Studie wurden das Gliptin und der SH in der Addon-Therapie bei 1035 mit mindestens 1500 mg/d Metformin unzureichend eingestellten Typ-2-Diabetikern verglichen. $\mathrm{lhr} \mathrm{HbA}_{1 \mathrm{c}}$ lag bei 6,5-9\%, 936 Patienten vollendeten die 32-wöchige Studie. Ihre Basistherapie und Begleitmedikationen behielten sie bei. Sie erhielten täglich 100 mg Sitagliptin ( $n=$ 516) oder nach der Titrierungsphase eine mittlere Dosis von 2,1 mg/d Glimepirid ( $n=519$ ) über 30 Wochen zusätzlich. Mit $100 \mathrm{mg} / \mathrm{d}$ Sitagliptin betrug die mittlere Veränderung des $\mathrm{HbA}_{1 c}-0,47 \%$-Punkte, mit $\mathrm{SH}$ waren es $-0,54 \%$-Punkte. Das Konfidenzintervall lag im vordefinierten Rahmen der Nichtunterlegenheit. $52,4 \%$ vs. 59,6\% der Patienten mit Gliptin bzw. SH erreichten bei Studienende einen $\mathrm{HbA}_{1 \mathrm{c}}$ $<7 \%,<6,5 \%$ war der $\mathrm{HbA}_{1 c}$ bei 21,2 bzw. $27,5 \%$. Der Nüchternblutzucker wurde vom Ausgangswert (Mittel 8 bzw. 8,1 mmol/l) mit Sitagliptin im Schnitt um 0,8, mit SH um $1 \mathrm{mmol} / \mathrm{l}$ verringert. Bei HDL und Triglyzeriden gab es Vorteile für die Gruppe mit Sitagliptin. Nur 7\% mit dem Gliptin, aber 22\% der mit $\mathrm{SH}$ behandelten Patienten berichteten von mindestens einer Hypoglykämie $(p<0,001)$. Patienten mit dem DPP-4-Hemmer hatten zudem ein geringeres Risiko wiederholter Hypoglykämien.

1. Arechavaleta R et al. Diabetes, Obesity and Metabolism 2011;13:160-8.

Quelle: MSD

\section{Antiadipositum mit neuem Namen}

Zur Unterstützung gewichtsreduzierender Maßnahmen steht das zentral wirksame Antiadipositum und indirekte Sympathomimetikum X 112 T der Riemser Arzneimittel AG seit 1. April 2011 unter dem neuen Handelsnamen Alvalin zur Verfügung. Der Wirkstoff Cathin (D-Norpseudoephedrin) mindert über eine Verstärkung von Noradrenalin im ZNS das Appetitgefühl. Bei Patienten mit mindestens 20-\%igem Übergewicht und ohne Empfehlung einer Ernährungseinschränkung führte die sechswöchige Gabe von Cathin zu einer Gewichtsabnahme von im Mittel 6,4 kg (Männer), und 7,9 kg (Frauen ) [1]. red

1. Szelenyi I et al. Wien Med Wochenschr 1974; 4: 49-52. Quelle: Riemser 